\title{
Ionic liquid-based vortex-assisted DLLME followed by RP-LC-PDA method for bioassay of daclatasvir in rat serum: application to pharmacokinetics
}

\author{
T. Siva Rao ${ }^{1 *}$, Marakada Sridevi ${ }^{1}$, Challa Gangu Naidu ${ }^{2}$ and Bandaru Nagaraju ${ }^{3}$
}

\begin{abstract}
Background: Daclatasvir is a direct-acting antiviral agent against hepatitis $\mathrm{C}$ virus (HCV) used for the treatment of chronic HCV genotype infections of 1 and 3. Hepatitis $C$ is an infectious liver disease caused by infection with hepatitis $C$ virus (HCV). There are no reports found to be daclatasvir in ionic liquid-based extraction.

Methods: A simple vortex assisted an environmental eco-friendly ionic liquid dispersive liquid-liquid microextraction method for determination of daclatasvir form rat serum. For the sample extraction, various green solvents, like ionic liquids, were used. The repercussion of various dispersive solvents, extractant, and disperser ratios was evaluated; non-identical ionic liquids assess the salt concentration on sample recoveries and enrichment factors were examined. Amid all the ionic liquids that were scrutinized, 1-butyl-3-methylimidazolium hexafluorophosphate was selected as the most effective ionic liquid.

Results: The present bioassay recoveries were found to be more than $99.4 \%$ at an extractant and disperser ratio of 0.43 with an addition of $5.0 \% \mathrm{NaCl}$ (sodium chloride) that was selected as an effective salt concentration for present extraction. Compared to protein precipitation, the enhanced detection and quantification limits attained were 0. $015 \mu \mathrm{g} / \mathrm{mL}$ and $0.045 \mu \mathrm{g} / \mathrm{mL}$, respectively. A linear relationship in the range of $0.05-10.0 \mu \mathrm{g} / \mathrm{mL}$ respectively with a correlation coefficient of $\left(r^{2}\right) 0.9996$ was observed.
\end{abstract}

Conclusion: The developed method was successfully applied to study the pharmacokinetics of daclatasvir in rat serum according to the bioanalytical method validation guidelines.

Keywords: Ionic liquid, Rat serum, Daclatasvir , Dispersive liquid-liquid microextraction, RP-LC-PDA

\section{Background}

Daclatasvir

(DCV,methylN-[(2S)-1-[(2S)-2-[5-(4' - \{2[(2S)-1-[(2S)-2-[(methoxycarbonyl)amino]-3-methylbutanoyl]pyrrolidin-2-yl]-1H-imidazol-5-yl\}-[1,1'-biphenyl]-4-yl)-1H-imidazol-2-yl] pyrrolidin-1-yl]-3-methyl-1oxobutan-2-yl] carbamate) is a direct-acting antiviral agent against hepatitis $\mathrm{C}$ virus (HCV) used for the treatment of chronic HCV genotype infections of 1 and 3. Hepatitis $C$ is an infectious (Anderson et al. 2004) liver disease caused by infection with hepatitis $C$ virus

\footnotetext{
* Correspondence: sivaraotvalluri.16@gmail.com

'Department of Inorganic and Analytical Chemistry, School of Sciences,

Andhra University, Visakhapatnam, India

Full list of author information is available at the end of the article
}

(HCV). The endogenous substances are present in the form of complex nature like blood fluids of different animals. Its hydrophilic molecule affinity (Zhang et al. 2009) is very high (complex biological fluids) in blood serum and plasma samples, and its high-extraction recovery (ER) contain sources, a novel technique for good laboratory practice. So, in such cases, it is necessary to develop rapid development techniques via (Vuckovic et al. 2010) protein precipitation (PP) method (Gaillard et al. 1995), solid phase extraction (SPE) method (Zeng et al. 2010), and liquid-liquid extraction (LLE) used to extract with eco-friendly solvents like IL resolves its complex nature with the effect of analytical and including biological matrices. Protein precipitation (PP) is 
routinely used methodologically for initial days for drug discovery and an efficient recovery owing to the rapid extraction procedure. However, it mainly resembles Lucie and Hana 2009 that developed a new bioanalytical sample extraction method, and Fu et al. 2005 reported a pharmaceutical method with acceptable specificity. Liquid-liquid extraction (LLE) is also an effective extraction methodology. Nuhu et al. 2011 described a new dispersive liquid-liquid extraction method via drugs exhibiting toxic nature which can perform hazardous nature in environmental samples. A novel by Rezaee et al. 2006 reported a microextraction technique for organic compounds. Berijani et al. 2006 determined a sensitive method for organophosphorous content in water samples, and Rezaee et al. 2010 reported a new microextraction method for biological samples.

DLLME is a fortunate extraction technique due to the high-contact surface of fine droplets of extractant solvent and analytes, which speeds up the mass-transfer processes of analytes. DLLME in its more classical variant has characteristics of cloud-point extraction, whereas some DLLME modes also resemble homogeneous liquid-liquid extraction. The method is useful because of its high-concentration factor, high-extraction efficiency, and minimum requirements for sample and organic solvents. The requirement of dispersive solvent is in the milliliter range (and so accompanied by sample dilution), and the necessity of a centrifugation step in order to facilitate the separation of the phases can be cited among the shortcomings. A novel by Jahromi et al. 2007 developed ultra-level of $\mathrm{Cd}$ in water samples. Nagaraju and Huang 2007 reported a triazine herbicides content in water samples. Berijani et al. 2006 determined an organophosphorus pesticides in water samples and (Kozani et al. 2007) quantification of chlorine content in ppm level. Determination of metal ions present in different extractable various materials (Fattahi et al. 2007; Rezaee et al. 2006; Shokoufi et al. 2007; Rahnama Kozani et al. 2007) like PAHs, chlorophenols, chlorobenzenes, organophosphorus pesticides, and triazine herbicides samples. However, compared to high-density organic solvents (Rezaee et al. 2009; Zhou et al. 2009) such as chlorobenzene, carbon tetrachloride, chloroform, and which are present in highly toxic in nature. Of late, room-temperature ILs (RTILs) are used as extraction solvents due to their eco-friendly nature and unique physicochemical properties. Latterly, a new extraction technique termed as temperature-controlled and vortex-assisted IL dispersive liquid-phase microextraction (DLLME) (Anderson et al. 2006) using 1-Hexyl-3methylimidazolium hexafluorophosphate was also tried for analysis of the few organophosphorus pesticides present in environmental samples. The recent applications of ILs in analytical chemistry were comprehensively reviewed and determined. Accordingly, the various investigations using ILs (Baker et al. 2005; Yao and Anderson 2009) for separation, enrichment, and extraction of traces of analytes from complex matrices with their bioassay has been increasing year by year and sofosbuvir and daclatasvir in human plasma have been determined. A derivitized microextraction method (Kramer and Andrews 2001) for 11-nor-delta9-tetrahydrocannabinol-9-carboxylic acid in urine samples, ion-pairing gas chromatographic method (Fiamegos and Stalikas 2007) for phenolic endocrine disruptors was done. Reactions of balofloxacin (Yan et al. 2006) were reported in serum samples. A validated assay method (Suzuki et al. 1995) for balofloxacin and daclatasvir in a tablet dosage form. A validated chiral-HPLC method (Srinivasu et al. 2016) and UV- spectrophotometric method (Jeevana Jyothy and Padmaja 2016) for daclatasvir. Bioanalytical literature survey reveals that few methods were published (Danielson et al. 2018; Rykowska et al. 2018; Bogdanov and Svinyarov 2018; Boli et al. 2018) in the field of sample extraction by using ILs to determination of drugs and their (Zhou et al. 2008; Liu et al. 2009; Nageswara Rao et al. 2014a, b, 2012) metabolites from urine samples.

The present manuscript illustrates the systematic development and validation of novel vortex-assisted IL-based DLLME method for daclatasvir (chronic hepatitis $\mathrm{C}$ virus $(\mathrm{HCV})$ ) genotype from rat serum. This is the first report on IL-based DLLME of daclatasvir from rat serum by vortex-assisted RP-HPLC method. To ensue the method, a series of dispersive solvents in different ratios of five different ILs and with the addition of different salt concentrations were tried. After the systematic enterprise, the present bioassay method was successfully extended to pharmacokinetic studies in rats.

\section{Methods}

\section{Availability of data and materials}

All the findings (chromatographic and pharmacokinetic) and data collected from Shri Vishnu Pharmacy College and VFSTR University, and materials purchased from Sigma-Aldrich. IL's and solvent delivery systems for extraction recovery and for separating solvents were of analytical grade. Ionic liquids viz., 1-butyl-3-methylimidazolium tetrafluoroborate (BMITB), 1-butyl-3-methylimidazolium hexafluorophosphate (BmimPF6), 1-butyl-3-methylimidazolium bromide (BMIB), (Fluka, Steinheim, Switzerland), 1-hexyl-3methylimidazolium chloride (HMIC), and 1-ethyl-3-methylimidazolium methyl sulfate (EMIMS), (Aldrich, Deisenhofen, Germany) $>96 \%$ of purity were used. HPLC grade methanol and acetonitrile $(\mathrm{MeOH})$ (Merck Fine chemicals, Mumbai, India), and acetic acid AR grade (SD Fine Chemicals, Mumbai, India) were used. High-purity water prepared by Millipore Milli-Q water purification system (Millipore 
synergy, Billerica, MA, USA), Membrane filter papers $(0.45 \mu \mathrm{m})$ (Whatmann, Sanford, FL, USA) were used.

\section{Animals and lab accessories}

Control blood used in the present study were collected from male Wistar rats (200-220 g) (Department of Pharmacology, Shri Vishnu College of Pharmacy, Vishnupur, Bhimavaram, India) housed under standard conditions and had ad libitum access to water and standard laboratory diet throughout the experiments and stored at $-20^{\circ} \mathrm{C}$ until the time for use. The multi-pipette for spotting blood (Tarsons, Kolkata, India), blood collection tubes (Sarstedt Leicester, UK), A centrifuge (model 2-16P, Sigma, Zurich, Switzerland), and heparin-coated capillaries (Sangius Counting Gmbh, Nümbrecht, Germany) were used. After a single dose by oral administration of $60 \mathrm{mg} / \mathrm{kg}$ of DCV to healthy Wistar rats $(n=6)$, blood samples $(0.2 \mathrm{ml})$ were collected into the processed test tubes at $0,0.5,1,2,3,4,5,6,7,8,9,10,11,12$, and $24 \mathrm{~h}$ post-dose. The blood samples were centrifuged at $5400 \times g$ for $10 \mathrm{~min}$, and the serum samples were stored at $-20^{\circ} \mathrm{C}$ until prior to analysis.

\section{High-performance liquid chromatography}

The HPLC equipment consisting of two LC-20AT pumps, SPD-M20A diode array detector, DGU-20A3 degasser, and CBM-20A system controller (all from Shimadzu, Kyoto, Japan) were used.

\section{Chromatographic data}

The chromatographic data were recorded using an HP-Vectra (Hewlett-Packard, Waldron, Germany) computer system with LC solution data-acquiring software (Shimadzu, Kyoto, Japan). Vortex Shaker, the sample tubes and repeater (Tarsons, Chennai, India), and centrifuge equipment (model 2-16P) (Sigma, Zurich, Switzerland) were used for the extraction and quantification of daclatasvir samples.

\section{Preparation of stock solutions}

Accurately weighed $10.0 \mathrm{mg}$ of each daclatasvir and ledipasvir (IS) (Fig. 1) were separately dissolved in $10.0 \mathrm{~mL}$ of methyl alcohol $(\mathrm{MeOH})$ in the ratio of $1 \mathrm{mg} / \mathrm{mL}$ in two volumetric flasks to give $1000 \mathrm{ppm}$ after preparation of stocks to prepare the diluted individuals stock solutions from and stored at $4{ }^{\circ} \mathrm{C}$ in a refrigerator prior to analysis.

\section{Preparation of calibration standards}

Calibration standards of ledipasvir were prepared by appropriate dilutions of stock solution with methyl alcohol $(\mathrm{MeOH})$ used as a diluent for all the vortex-assisted samples. The working standards (WS) were used for the preparation of whole serum calibration standards (range $0.04-10 \mu \mathrm{g} / \mathrm{mL}$ ) were prepared by spiking working standard solutions to serum taken in Eppendorf tubes. These calibration standards were used to validate all the chromatographic parameters. All the calibration standards were stored at $4{ }^{\circ} \mathrm{C}$ in a refrigerator prior to analysis.

\section{Preparation of quality control samples}

The quality control samples (QCs) $(0.05,0.1,2.0,5.0$, and $7.5 \mu \mathrm{g} / \mathrm{mL}$ ) were prepared by spiking $100 \mu \mathrm{L}$ of suitable working standards (WS) to $200 \mu \mathrm{L}$ of extracted serum samples. Quality control (QC) samples were stored at $4{ }^{\circ} \mathrm{C}$ in a refrigerator prior to analysis. The QC samples were used to evaluate enrichment factors (EF) and extraction recovery (ER).

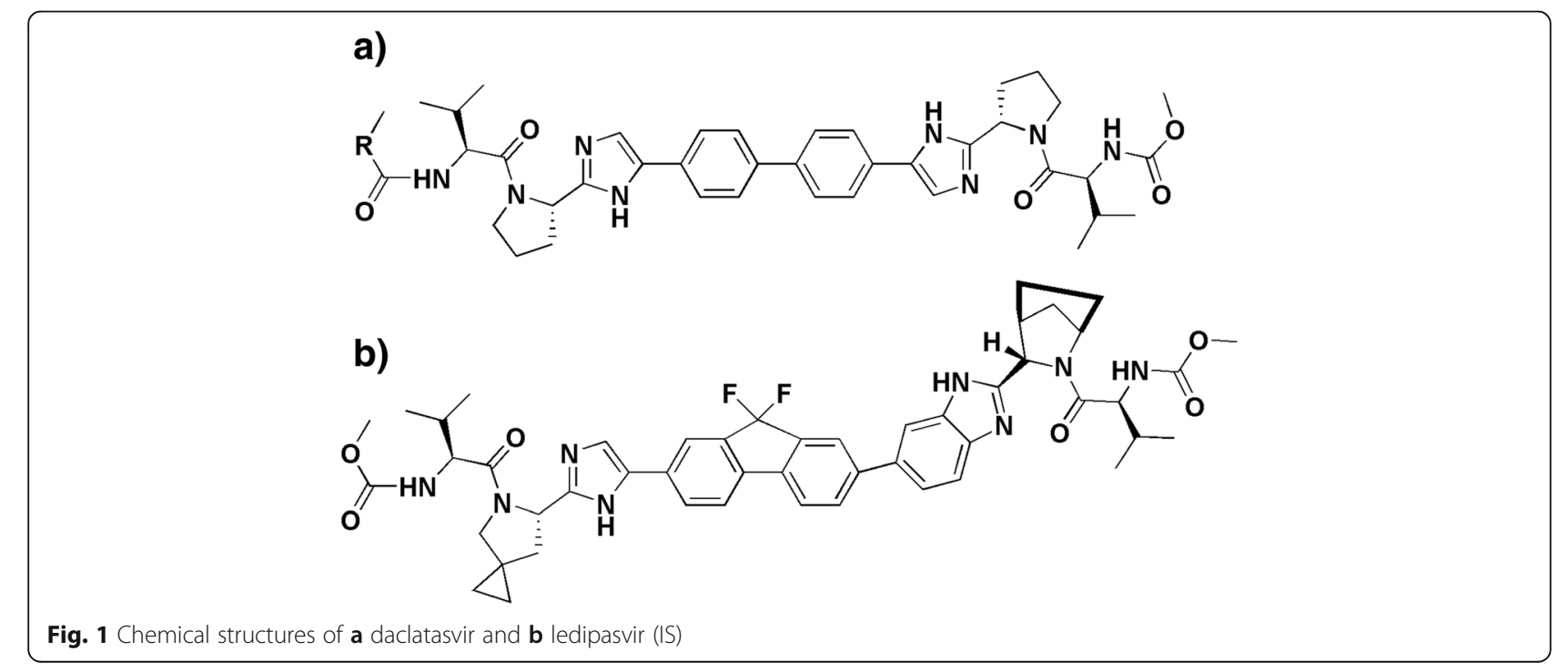




\section{Systematic extraction of daclatasvir from rat serum sample}

The extraction was executed in Eppendorf tubes, which are accessible for sample collection. The tubes were simply filled with quality control (QC) samples followed by addition of IS, ILs of (BMITB//BMIB/HMIC/EMIMS/ BmimPF6/ $\left.\mathrm{C}_{6} \mathrm{MIMPF}_{6}\right)$, Acetonitrile $(\mathrm{ACN})$, and concentrated $5 \% \mathrm{NaCl}$ salt solutions $(60 \mu \mathrm{L})$. After addition of IL, the mixture was vortexed for $2 \mathrm{~min}$ (commonly assisted for all the samples) and centrifuged at $5400 \times g$ speed for $5.0 \mathrm{~min}$ interval time. After relaxation time, the bottom layer which is the colored area, consisting of IL, was collected using a long microsyringe with a rubber septum and injected directly on to Waters Xterra $\mathrm{C}_{18}$ column for analysis. The block diagram showing the IL-DLLME of daclatasvir from rat serum, the systematic procedure is shown in Additional file 1: Figure S1. In case of in vitro samples, $200 \mu \mathrm{L}$ of the serum spiked with $100 \mu \mathrm{L}$ of daclatasvir was taken in a $1.0-\mathrm{mL}$ centrifuge tube (Eppendorf) with the help of Tarson's micro pipette along with the suitable microtip. In this, $60 \mu \mathrm{L} A C N$, $20 \mu \mathrm{L}$ IS, $60 \mu \mathrm{L} 5 \% \mathrm{NaCl}$ solution, and $40 \mu \mathrm{L}$ of IL were added then vortexed for $2 \mathrm{~min}$ and centrifuged at $5400 \times g$ for $5.0 \mathrm{~min}$. The IL was resolved on the bottom of Eppendorf to be separated from the serum sample. The IL was originally colorless, but after some time, it turned into yellow due to the transfer of daclatasvir whose color was yellow which is shown in Eppendorf tube (extract). The IL was taken carefully from the Eppendorf tube using a microsyringe and injected directly on to RP-LC Waters Xterra $C_{18}$ column. The probable interaction of DCV with serum and IL, mechanism of phase separation of IL, and rat serum containing daclatasvir are shown in Additional file 1: Figure S2. In case of in vivo samples, $200 \mu \mathrm{L}$ of the serum was directly mixed with $40 \mu \mathrm{L}$ IL, $60 \mu \mathrm{L} \mathrm{ACN}, 20 \mu \mathrm{L}$ IS, and $5 \%$ $\mathrm{NaCl}$ salt solution. The mixture was vortexed and assisted as validated for $2 \mathrm{~min}$ and followed by centrifugation at $5400 \times g$ for $5.0 \mathrm{~min}$. The remaining procedure was similar as in case of in vitro samples. The schematic representation of IL-based DLLME of daclatasvir from rat serum samples is shown in Fig. 2.

\section{Chromatographic conditions}

After many systematic trails, the chromatographic separation was accomplished on Waters Xterra $C_{18}$ column $(250 \times 4.6 \mathrm{~mm} ; 5 \mu \mathrm{m})$ under an isocratic mode of elution. The mobile phase was a mixture of $10 \mathrm{mM}$ ammonium acetate: acetonitrile: methanol (65:20:15, $v / v / v)$. It was freshly prepared, filtered through a Millipore filter (pore size $0.45 \mu \mathrm{m}$ ), and degassed continuously by an online degasser and sonicated for 25 min prior to pumping system for analysis. The separation was achieved at room temperature using a 1.0 $\mathrm{mL} / \mathrm{min}$ flow rate and $12 \mathrm{~min}$ run time. The injection volume was $20 \mu \mathrm{L}$, and the detection wavelengths were set at $305 \mathrm{~nm}$. The chromatographic and the integrated data were recorded by using an HP-Vectra (Hewlett-Packard, Waldron, Germany) computer system using LC-Solution data-acquiring software (Shimadzu, Kyoto, Japan) were used.

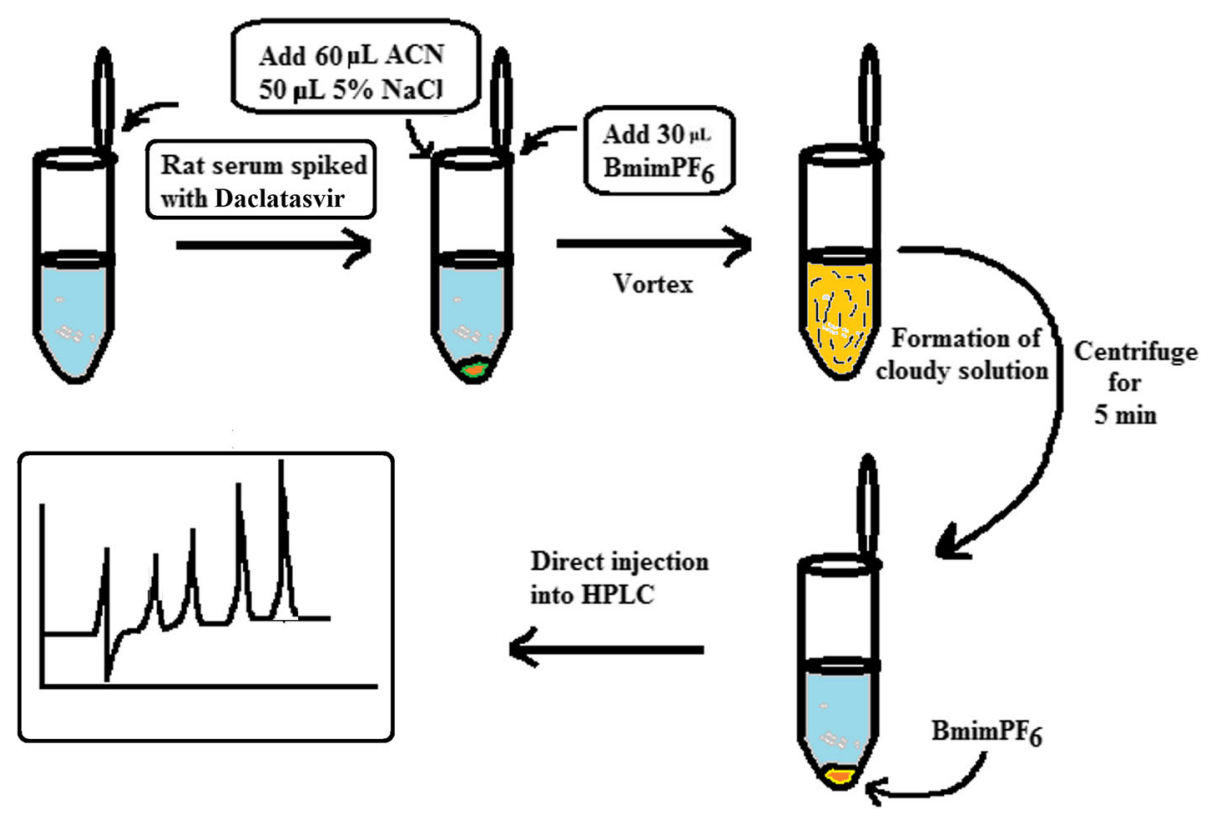

Fig. 2 Schematic representation of vortex-assisted an IL-based DLLME of daclatasvir 


\section{Analytical parameters}

The present vortex-assisted bioassay accord the analytical parameters, although we focused on two important parameters, i.e., enrichment factor (EF) and extraction recovery (ER) were determined for evaluation of the recommended vortex assisted assay method. ER was defined as the percentage of daclatasvir extracted into IL calculated by Eq. (1), ER $=\left(C_{i} \times V_{i} \times 100\right) /\left(C_{0} \times V_{0}\right)(1)$ where $C_{i}$ and $C_{0}$ are the concentration of daclatasvir in the IL and the initial concentration of the rat serum, respectively. $V_{i}$ and $V_{0}$ are the volumes of the phases separated in the sample. The EF was defined as the ratio of the concentration of daclatasvir in IL to the initial concentration of the sample. EF was calculated by Eq. (2), $\mathrm{EF}=C_{i} / C_{0}(2)$ respectively for current bioassay.

\section{Results and discussion}

\section{Optimization of the extraction conditions}

The developed validated vortex assisted bioassay extraction conditions are optimized by using systematically. The adverse application of ILs to DLLME of blood serum samples through its isolation methodology mainly depends on two factors (i) extraction recovery of IL from rat serum and (ii) the IL/dispersive solvent ratio measured and miscibility/interaction of IL with serum plays a foremost role in its extraction recovery for current bioassay.

\section{DLLME procedure and selection of ionic liquid}

The systematic dispersive liquid-liquid microextraction (DLLME) has the most outcoming advantages of rapidity, low sample volume, low cost, high-extraction recovery, simplicity, and a high-enrichment factor over the traditional methods. In DLLME, it is considerable to select an extraction solvent and also elect the dispersive ratio of individual ratio of solvent front from reservoirs. In other cases, toxic solvents such as tetrachloroethylene, carbon disulfide and chlorobenzene, and carbon tetrachloride have been openly used as extraction solvents for current bioassay. In order to reduce the effect of toxic solvent on the environment, eco-friendly solvents like ILs are expected to be used. Room temperature ILs (RTILs) are generally newly appealed ILs, known as novel solvents, have been widely used in separation and other extraction fields and shows properties like excellent solubility, include low volatility, chemical, and thermal stability nature.

Table 1 Various ILs and their properties used in vortex-assisted DLLME

\begin{tabular}{|c|c|c|c|c|c|}
\hline Name of IL & Code & Cation & Anion & $\begin{array}{c}\text { Miscibility } \\
\text { with rat } \\
\text { serum }\end{array}$ & $\begin{array}{l}\text { Density } \\
(\mathrm{g} / \mathrm{mL}) \\
\text { at } 20^{\circ} \mathrm{C}\end{array}$ \\
\hline $\begin{array}{c}\text { 1-Butyl-3- } \\
\text { methylimidazolium } \\
\text { tetrafluoroborate }\end{array}$ & BMITB & & & partial & 1.21 \\
\hline $\begin{array}{c}\text { 1-butyl-3- } \\
\text { methylimidazolium } \\
\text { bromide }\end{array}$ & BMIB & & $\mathrm{Br}^{-}$ & Miscible & 1.30 \\
\hline $\begin{array}{l}\text { 1-butyl-3- } \\
\text { methylimidazolium } \\
\text { hexaflurophosphate }\end{array}$ & $\mathrm{BmimPF}_{6}$ & & & Immiscible & 1.38 \\
\hline $\begin{array}{l}\text { 1-Hexyl-3- } \\
\text { methylimidazolium } \\
\text { hexaflurophosphate }\end{array}$ & $\mathrm{C}_{6} \mathrm{MIMPF}_{6}$ & & & partial & 1.41 \\
\hline $\begin{array}{l}\text { 1-hexyl-3- } \\
\text { methylimidazolium } \\
\text { chloride }\end{array}$ & HMIC & & $\mathrm{Cl}^{-}$ & Miscible & 1.05 \\
\hline $\begin{array}{c}\text { 1-ethyl-3- } \\
\text { methylimidazolium } \\
\text { methylsulfate }\end{array}$ & EMIMS & & & partial & 1.28 \\
\hline
\end{tabular}


The ILs viz., HMIC/EMIMS/BMITB/BmimPF6/ $\mathrm{C}_{6} \mathrm{MIMPF}_{6 /} \mathrm{BMIB}$ were originally selected to check their appropriateness and their physical behavior like MPs, BPs, and density of each IL are studied and data of ILs are given in Table 1. After several observations of various extracts, we found that HMIC and BMIB have shown complete miscibility indicating their strong interactionlaffinities on rat serum samples. The remaining ILs and $\mathrm{C}_{6} \mathrm{MIMPF}_{6}$, EMIMS, and BMITB have shown partially miscibility with similar serum samples. Based on the above scrutiny, the IL for current bioassay was finalized. So, the $\mathrm{C}_{6} \mathrm{MIMPF}_{6}$ (1-Hexyl-3-methylimidazolium hexafluorophosphate) was eliminated and finally BmimPF6 (Omitted remaining IL's) does not show interaction with proteins with high extraction recoveries. So, finally BmimPF6 IL was found to be a suitable extraction solvent for the current vortex-assisted bioassay.

\section{Selection of dispersive solvent}

A systematic vortex-assisted extraction procedure is displayed in Additional file 1: Figure S3. The choice of a dispersive solvent (DS) with suitable miscibility in both IL and serum sample phase (which is present in Eppendorf) plays the main role because of the fact that the IL could completely disperse into the aqueous phase of serum used as a disperser is an optimized solvent. In the present core, the investigation is mainly focused on solvents, such as acetone, ACN, and ethanol, that were tried for extraction for bioassay, as they fulfilled both the extracts. Partition coefficients of daclatasvir were high in ACN (solvent) as shown in Fig. 3a it was optimized as a dispersive solvent (DS) (Table 1). The remaining mixtures have an ACN as a dispersive solvent (DS) and BmimPF6 (IL) as an extractant in different volume ratios that were chosen for current bioassay for remaining all the extracts.

\section{a)}

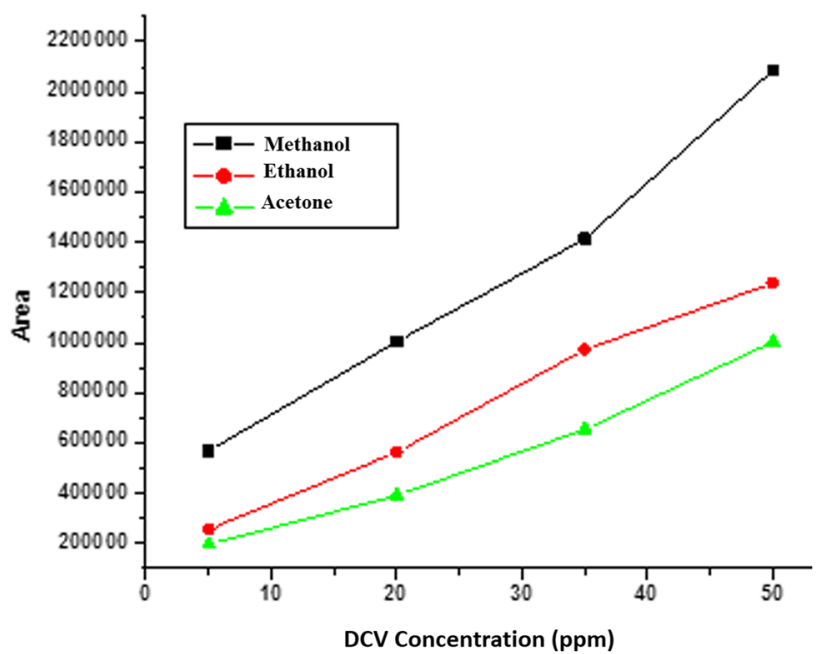

b)

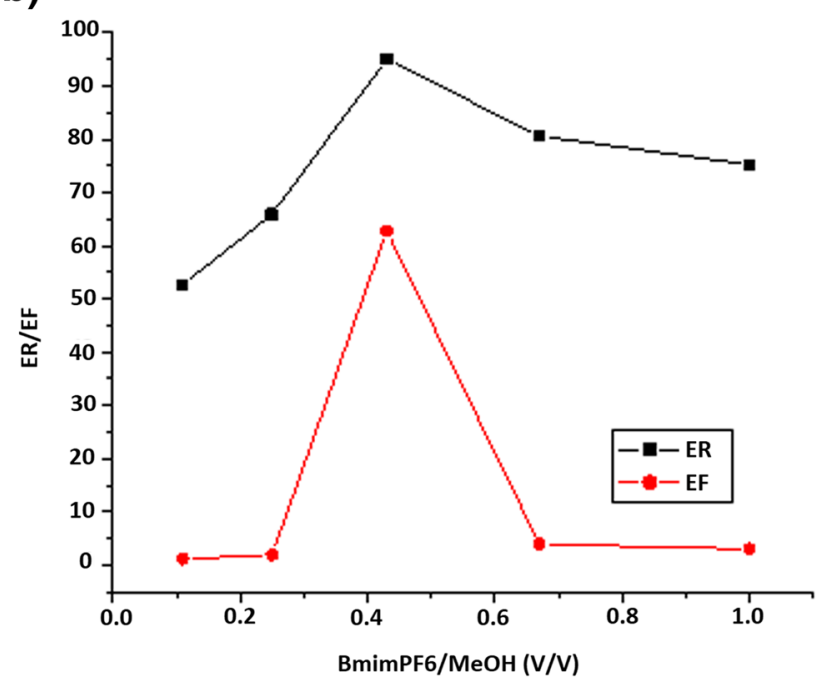

c)

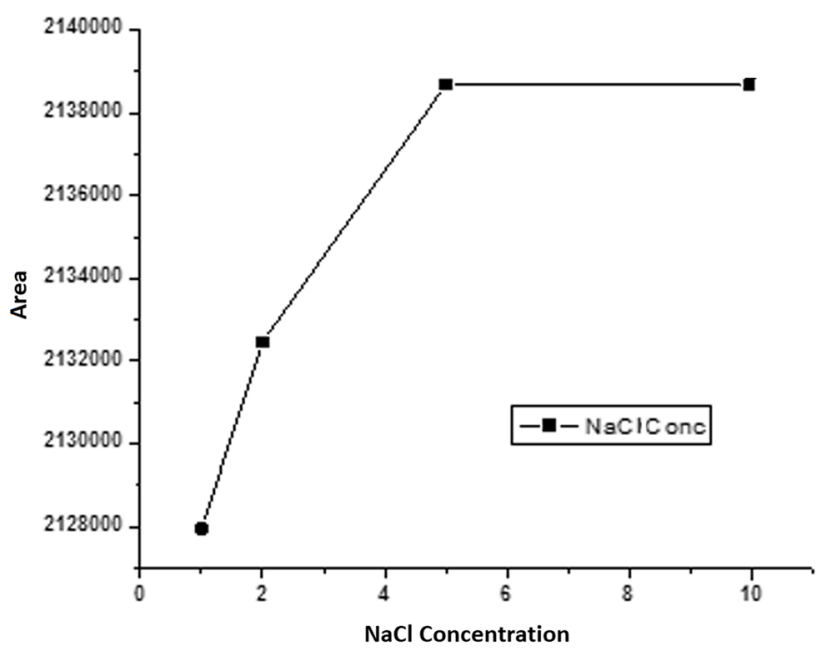

Fig. 3 Effects of various parameters a dispersive solvents, b BmimPF6: ACN ratio, and $\mathbf{c} \% \mathrm{NaCl}$ on the extraction of daclatasvir 
Table 2 Partition ratio and recovery (\%) of daclatasvir ${ }^{a}$ at different IL:ACN ratios and $\mathrm{NaCl}$ concentrations

\begin{tabular}{|c|c|c|c|c|c|c|}
\hline \multirow[t]{2}{*}{ Conditions } & \multicolumn{2}{|c|}{ Concentration of daclatasvir (mM) } & \multicolumn{2}{|l|}{ Peak area } & \multirow{2}{*}{$\begin{array}{l}\text { Partition } \\
\text { ratio }(p)\end{array}$} & \multirow{2}{*}{$\begin{array}{l}\text { Recovery } \\
(\%)\end{array}$} \\
\hline & $\mathrm{IL}$ & Serum & $\mathrm{IL}$ & Serum & & \\
\hline \multicolumn{7}{|l|}{ IL:ACN (V/V) } \\
\hline 0.11 & 0.0435 & 0.0301 & 1151275 & 1005199 & 1.447 & 52.7 \\
\hline 0.25 & 0.0524 & 0.0245 & 1426919 & 724542 & 2.138 & 65.9 \\
\hline 0.43 & 0.0720 & 0.0022 & 2118122 & 30648 & 32.72 & 98.8 \\
\hline 0.67 & 0.0627 & 0.0175 & 1703875 & 447811 & 3.582 & 80.6 \\
\hline 1.00 & 0.0560 & 0.0195 & 1593382 & 559283 & 2.971 & 75.2 \\
\hline \multicolumn{7}{|l|}{$\mathrm{NaCl}(\%)$} \\
\hline 1.0 & 0.0817 & 0.0010 & 2129955 & 20739 & 81.7 & 99.1 \\
\hline 2.0 & 0.0745 & 0.0008 & 2127401 & 15840 & 93.1 & 99.0 \\
\hline 5.0 & 0.0712 & 0.0006 & 2130342 & 10811 & 118.6 & 99.5 \\
\hline 10.0 & 0.0704 & 0.0006 & 2130342 & 10811 & 118.6 & 99.5 \\
\hline
\end{tabular}

${ }^{\text {a Spiked concentration }=0.0630 \mathrm{mM}}$

\section{Optimization of extractant/disperser ratio}

The specific IL volume recovered in serum samples and the disperser/extractant volume ratio was optimized based on their EFs and ER values. The systematic extraction procedure (Fig. 3b) and the partition coefficient and extraction recovery values are documented in Additional file 1: Table S1. It could be seen from the results (Additional file 1: Table S1 and Fig. $3 \mathrm{~b}$ ) the maximum values at a dispersive ratio of 0.43 for current vortex-assisted bioassay.

\section{Effect of extraction and centrifugal time}

In vortex-assisted DLLME procedure from rat serum sample, the effect of extraction time was explored between 0.0 and $10.0 \mathrm{~min}$ and was observed that there was no considerable effect after 1 min of the extraction time on the efficiency of the serum extract. The mass transfer of both the (DCV and ledipasvir (IS)) analytes could slow down in Eppendorf, due to its more viscosity of ILs, but IL-DLLME reaches equilibrium. So, after injection of ACN and IL into rat serum sample extract, the mixture was vortex assisted for $2 \mathrm{~min}$. Centrifugation is a pivotal step in the DLLME for all the extractions that were achieved by varying various centrifugation time intervals that was observed from 2.0 to $14.0 \mathrm{~min}$ at divergent mean interval time $3.0 \mathrm{~min}$. The obtained results showed that the volume of the IL phase enhanced from 28.5 to $29.5 \mu \mathrm{L}$ levels by increasing centrifugal time from 2.0 to $5.0 \mathrm{~min}$ intervals each consecutive runs from effective

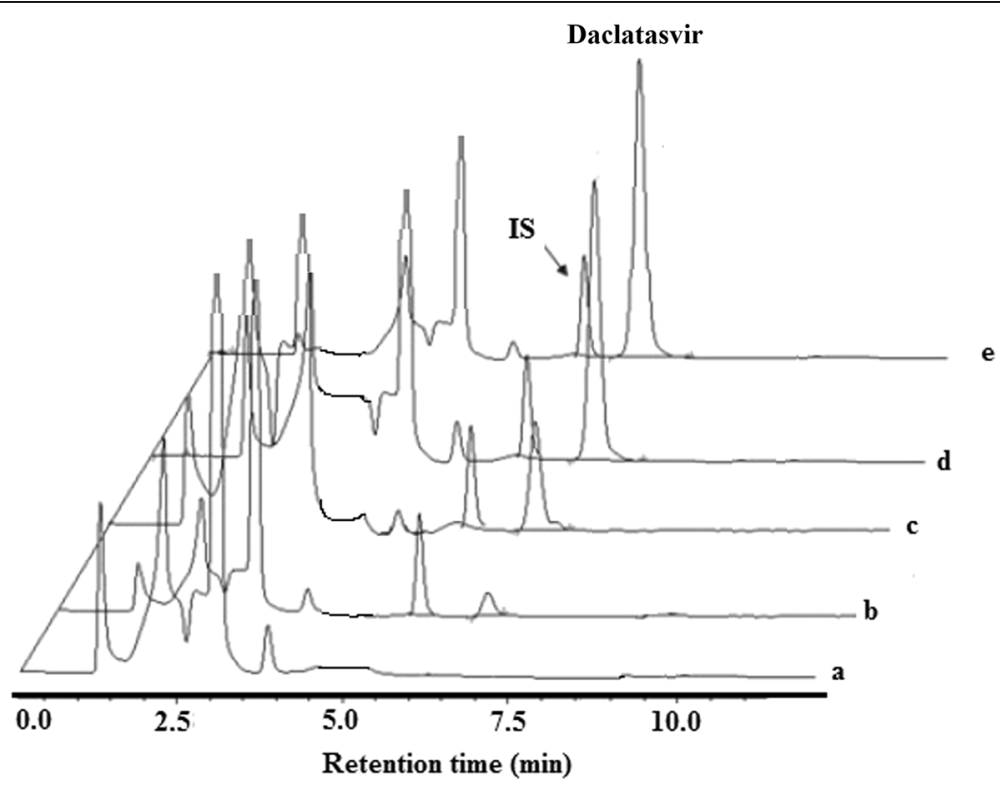

Fig. 4 Typical representative chromatograms of IL-DLLME extracts of rat serum spiked with daclatasvir at different concentrations a blank, b 0.1 , c 1.0 , d 5.0 , and e $10.0 \mathrm{ppm}$ 


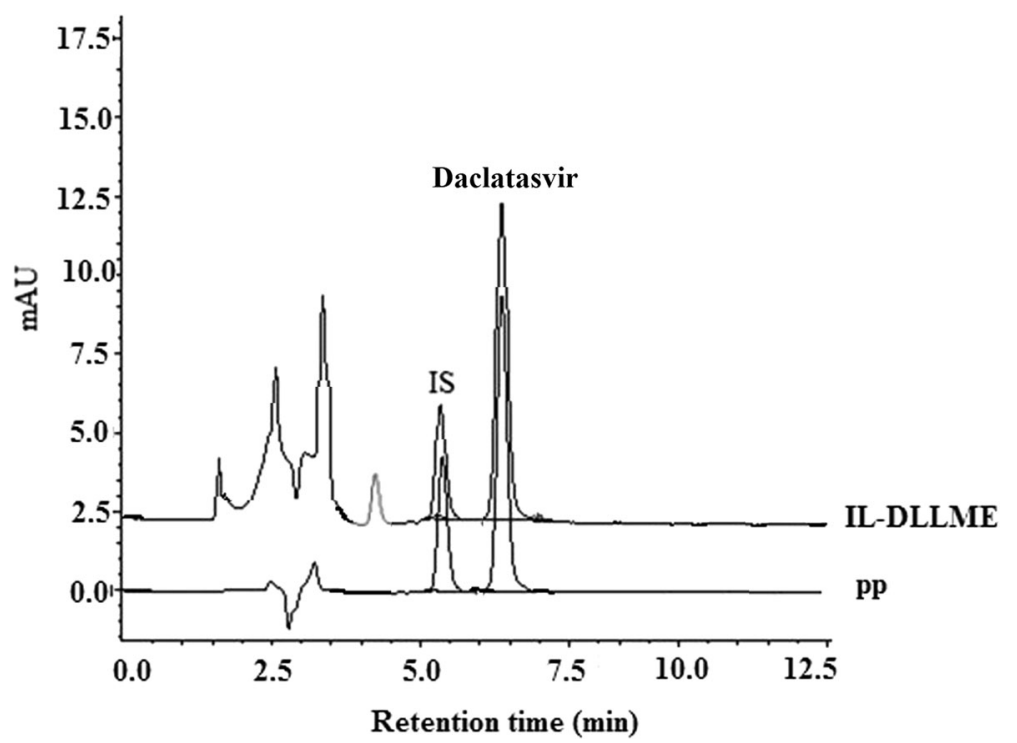

Fig. 5 Typical representative chromatograms of protein precipitation (PP) and IL-DLLLME extracts of daclatasvir from rat serum

extraction. Finally, 5.0 min centrifugal time was selected as an optimum condition for current vortex-assisted bioassay.

\section{Effect of sodium chloride (salt) on extraction recovery}

Sodium chloride (salt) impacts the current bioassay extraction efficiency, and it is shown (Fig. 3c) by the addition of salt concentrations levels are $1-10 \%(w / v)$ examined to the rat serum at the optimum experimental conditions from the extracted rat serum samples. After examining the results, there was a significant enhancement in partition ratio values (significance) as shown in Table 2. It could be seen from Table 2 that the supreme (most effective) recovery (99.5\%) of daclatasvir was found to be at $5 \%(w / v) \mathrm{NaCl}$ (sodium chloride) concentration level.

\section{Analytical performance of the method}

Analytical performance of the validated assay method can be determined in the terms of accuracy, precision, relative recovery, and stability of the sample. The optimized vortex-assisted, an IL-based DLLME method, was determined by examining both the extraction parameters (EF and ER) and the analytical figures of merit. Additional file 1: Table S2 reviewed the EF and ERs of daclatasvir concentrations at different linear levels $(0.05,0.1$, 2.05 .0 , and $7.5 \mu \mathrm{g} / \mathrm{mL}$ ) deliberated under the optimal conditions. The statistical data of both the parameters were evaluated at multi concentration levels. The EFs varied between 10.1 and 10.8, while the ERs varied between 98.4 and $99.5 \%$ respectively. The obtained results are given in Additional file 1: Table S2. The calibration curve was drawn by plotting the ratio of the daclatasvir and ledipasvir (IS) peak areas against concentration of daclatasvir concentration. Working standards (WS) containing daclatasvir at five different concentration levels in the range of $0.05-10 \mu \mathrm{g} / \mathrm{mL}$, (representative linear regression equation $y=34.291 x+43.064$ ) and the correlation coefficient was 0.9996 . The collected extracts of various concentrations levels of daclatasvir with ledipasvir (IS) were eventually analyzed by RP-LC-PDA method. The use of ledipasvir as an internal standard (IS) determine the daclatasvir concentration in order to minimize or reduce the matrix interferences from biological sample extract.

The representative chromatograms are shown in Fig. 4. The chromatograms of blank and spiked serum extracted sample indicate that there was no significant interference from blank (IL) peaks (from chromatogram). The detection levels were also increased when compared with protein precipitation (PP) method displayed in Fig. 5. Results indicate that (calculated statistical data) good linearity observed between the corresponding peak area and the concentration of the sample was obtained and its correlation coefficient $\left(R^{2}>0.999\right)$. Limit of detection (LOD) was calculated according to $\mathrm{S} / N=3$ ratio shows as $0.015 \mu \mathrm{g} / \mathrm{mL}$ for current bioassay. Limit of quantification (LOQ) also calculated according to $S / N=10$ ratio shows $0.045 \mu \mathrm{g} / \mathrm{mL}$ data

Table 3 Validation data

\begin{tabular}{ll}
\hline Parameter & Obtained value \\
\hline Linear range $(\mu \mathrm{g} / \mathrm{mL})$ & $0.05-10$ \\
LOD $(\mu \mathrm{g} / \mathrm{mL})$ & 0.015 \\
$\operatorname{LOQ}(\mu \mathrm{g} / \mathrm{mL})$ & 0.045 \\
RSD $(\%)$ & $<5.0$ \\
RR $(\%)$ & 99.5
\end{tabular}

$L O D$ limit of detection, $R S D$ relative standard deviation, $R R$ relative recovery 
Table 4 Stability data of daclatasvir from rat serum

\begin{tabular}{|c|c|c|c|}
\hline Storage conditions & Added conc. $(\mu \mathrm{g} / \mathrm{mL})$ & Calculated conc. $(\mu \mathrm{g} / \mathrm{mL})($ mean \pm S.D) & RE (\%) \\
\hline \multirow[t]{5}{*}{ Ambient (6h) } & 0.05 & $0.07 \pm 1.92$ & 2.4 \\
\hline & 0.1 & $0.12 \pm 2.34$ & 2.9 \\
\hline & 2.0 & $1.95 \pm 3.02$ & 2.5 \\
\hline & 5.0 & $2.95 \pm 3.01$ & 2.3 \\
\hline & 7.5 & $7.52 \pm 3.83$ & 3.5 \\
\hline \multirow[t]{5}{*}{3 freeze $\left(-20^{\circ} \mathrm{C}\right)$ thaw cycles } & 0.05 & $0.04 \pm 3.07$ & 2.1 \\
\hline & 0.1 & $0.09 \pm 4.41$ & 2.6 \\
\hline & 2.0 & $2.05 \pm 3.80$ & 3.1 \\
\hline & 5.0 & $2.90 \pm 3.02$ & 2.4 \\
\hline & 7.5 & $7.48 \pm 4.29$ & 4.2 \\
\hline \multirow[t]{5}{*}{$2-10^{\circ} \mathrm{C}, 3$ days } & 0.05 & $0.06 \pm 3.54$ & 2.9 \\
\hline & 0.1 & $0.13 \pm 4.07$ & 3.8 \\
\hline & 2.0 & $1.96 \pm 3.45$ & 2.4 \\
\hline & 5.0 & $2.90 \pm 3.07$ & 2.4 \\
\hline & 7.5 & $7.42 \pm 4.21$ & 3.7 \\
\hline \multirow[t]{5}{*}{ Autosampler stability (24 h) } & 0.05 & $0.08 \pm 2.90$ & 3.2 \\
\hline & 0.1 & $0.15 \pm 3.42$ & 2.9 \\
\hline & 2.0 & $2.10 \pm 2.61$ & 2.2 \\
\hline & 5.0 & $2.91 \pm 3.03$ & 2.4 \\
\hline & 7.5 & $7.44 \pm 4.22$ & 3.8 \\
\hline
\end{tabular}

$S . D$ standard deviation, $R E$ relative error $(n=6)$

are disposed in Table 3, and the intra-day and inter-day assay variation data are given in Additional file 1: Table S3.

\section{Stability data of daclatasvir}

Daclatasvir stability was performed by evaluating the different examine conditions were checked from rat serum extracts during the storage conditions. Stability experiments were performed (at Ambient, QC, freeze-thaw, autosampler) at five different concentration levels $(0.05,0.1,2.0,5.0$, and $7.5 \mu \mathrm{g} / \mathrm{mL}$ ) in triplicate ranges are drawn and pinched. Daclatasvir was considered to be stable in stock, as well as working solutions (WS) having the mean recoveries are found to be in the range 98.4-99.5\% of the original concentration values, which is compared to the biological sample

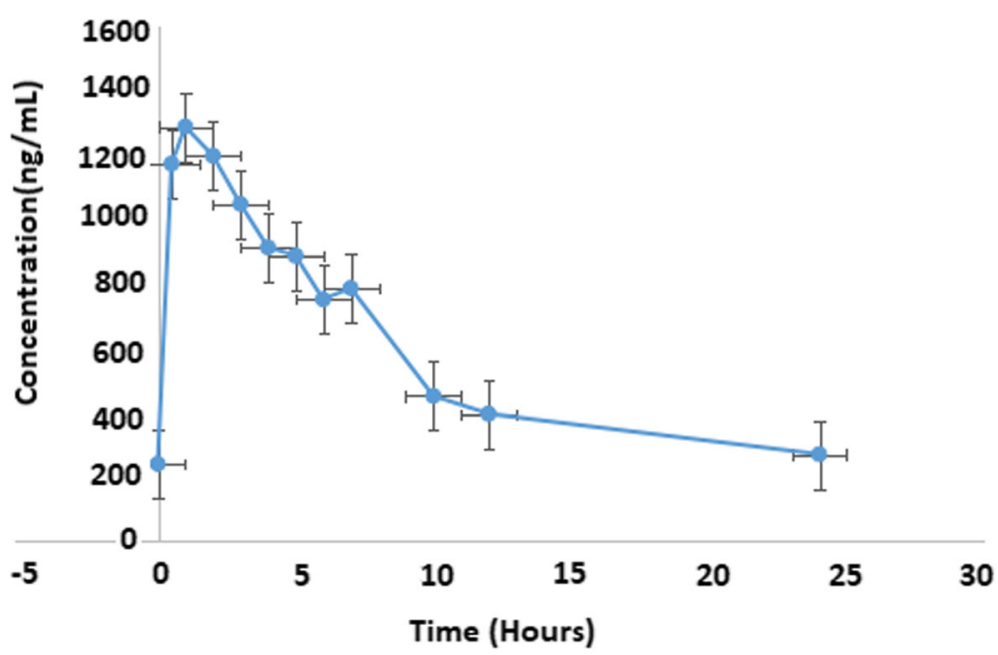

Fig. 6 Concentration vs. time profile over $24 \mathrm{~h}$ of daclatasvir in the serum samples $(n=6)$ by the administration of receiving a single $60 \mathrm{mg} / \mathrm{kg}$ dose of daclatasvir 
Table 5 Pk data of daclatasvir

\begin{tabular}{ll}
\hline Parameter DCV & \\
\hline$t_{\max }(\mathrm{h})$ & 1.62 \\
$C_{\max }(\mathrm{ng} / \mathrm{ml})$ & 1306.15 \\
$A \cup C_{\mathrm{o}-\mathrm{t}}(\mathrm{ng} / \mathrm{ml} / \mathrm{h})$ & 15465.4 \\
$\mathrm{~A} \cup C_{\mathrm{o}-\mathrm{a}}(\mathrm{ng} / \mathrm{ml} / \mathrm{h})$ & 15685.5 \\
$\mathrm{t}_{1 / 2}(\mathrm{~h})$ & 11.07
\end{tabular}

$C_{\max }(\mathrm{ng} / \mathrm{mL})$ maximum plasma concentration, $t_{\max }$ time to $C_{\text {max }} A U C$ area under the plasma concentration-time curve, $t_{1 / 2}$ half-life

matrices recoveries that were found to be $86.0-99.5 \%$ respectively. The results are given in Table 4 .

\section{Application to pharmacokinetic studies in rats}

The present bioassay method was successfully used for determination and quantification of daclatasvir concentration in rat serum after oral administration of prescribed conditions. The schematic representative concentration-time profile of daclatasvir is presented in Fig. 6. It is indicated that the daclatasvir concentration was found to be less than $0.5 \mu \mathrm{g} / \mathrm{mL}$ at initial (time intervals) hours of dosage, and the rate of absorption via drug-serum interaction was limited to poor nature in an early time interval. But, after 60 min, the concentration tends to show maximum absorption and then gradually diminish up to 1 day time interval; data are given in Table 5. The maximum absorbance of daclatasvir in rat serum sample was found to be $1.295 \mu \mathrm{g} / \mathrm{mL}$ respectively, so finally, the validated bioassay is successfully applied to pk studies in rats.

\section{Conclusions}

The present IL-based DLLME by RP-LC-PDA bioassay method is simple, reproducible, and an environmental eco-friendly; investigation explores for the determination and quantification of daclatasvir that was evaluated for the first time in a novel combination of vortex-assisted method and powerful preconcentration microextraction-based technique for extraction of daclatasvir from rat serum. The extraction time was done in a very short period of time, because of enormous surface area contact between the analyte and serum extraction solvent. Some of the advantages of this technique including the following: (i) compatibility with RP-LC-PDA method; (ii) no time-consuming procedures after extraction for the cleanup procedure and reducing the volume of extracted solution in the serum extract (same interval); and (iii) an environmental eco-friendly green solvent. The performance of the tender method in the extraction of daclatasvir from rat serum sample was found to yield magnificent results and successfully applied to pk studies in rats.

\section{Additional file}

Additional file 1: Figure S1. The schematic diagram showing the IL-DLLME
process of daclatasvir from rat serum. Figure S2. The systematic predictable
mechanism and its phase separation of IL from rat serum. Figure S3.
Optimization of vortex-assisted an IL-based DLLME conditions. Table S1. Partition
ratio and (\%) recovery of daclatasvir ${ }^{a}$ into IL. Table S2. Extraction recovery (ER)
and enrichment factor (EF) of daclatasvir. Table S3. Intra- and inter-day assay
variation data. (DOCX $6487 \mathrm{~kb}$ )

\section{Abbreviations}

ACN: Acetonitrile; BmimPF6: 1-butyl-3-methylimidazolium hexafluorophosphate; DLLME: Dispersive liquid-liquid microextraction; EF: Enrichment factor; ER: Extraction recovery; HCV: Hepatitis C virus; IL: Ionic liquid; IS: Internal standard; LLE: Liquid-liquid extraction; MeOH: Methanol; PP: Protein precipitation; RTILs: Room-temperature ionic liquids; WS: Working standard

\section{Acknowledgements}

The authors would like to thank AU \& Vignan's University for encouragement and making valuable suggestions to communicate the manuscript for publication and special thanks to SVCP for sample extraction and pharmacokinetic data collection.

\section{Funding}

There are no financial sources reported.

\section{Authors' contributions}

TSR and CGN conceived the main idea and implementation of the work by selecting the drug and wet lab analysis. SM and NR extract the biosamples and analyzed using vortex-assisted RP-HPLC methods. SM performed the wet lab study of collecting and diluting of the sample after validating the conditions for the drug sample. NR also helped in the mobile phase preparation during the HPLC study. All authors read and approved the final manuscript.

\section{Competing interests}

The authors declare that they have no competing interests.

\section{Publisher's Note}

Springer Nature remains neutral with regard to jurisdictional claims in published maps and institutional affiliations.

\section{Author details}

${ }^{1}$ Department of Inorganic and Analytical Chemistry, School of Sciences, Andhra University, Visakhapatnam, India. Vignan's Foundation For Science, Technology and Research (Deemed to be University), Vadlamudi, Guntur, Andhra Pradesh 522213, India. ${ }^{3}$ Department of Pharmacology, Shri Vishnu College of Pharmacy, Vishnupur, Bhimavaram 534202, India.

Received: 16 October 2018 Accepted: 10 April 2019

Published online: 29 April 2019

\section{References}

Anderson JL, Armstrong DW, Wei GT. Ionic liquids in analytical chemistry. Anal Chem. 2006;78(9):2892-4.

Anderson NL, Polanski M, Pieper R, Gratlin T, Tirumalai RS, Conrads TP, Veenstra TD, Adkins JN, Pounds JG, Fagan R, Lobley A. The human plasma proteome: a nonredundant list developed by combination of four separate sources. $\mathrm{Mol}$ Cell Proteomics. 2004;3(4):311-26.

Baker GA, Baker SN, Pandey S, Bright FV. An analytical view of ionic liquids. Analyst. 2005;130:800-8.

Berijani S, Assadi Y, Anbia M, Hosseini MRM, Aghaee E. Dispersive liquid-liquid microextraction combined with gas chromatography-flame photometric detection. Very simple, rapid and sensitive method for the determination of organophosphorus pesticides in water. J Chromatogr A. 2006;1123(1):1-9.

Bogdanov MG, Svinyarov I. Efficient purification of halide-based ionic liquids by means of improved apparatus for continuous liquid-liquid extraction. Sep Purif Technol. 2018;196:57-60.

Boli E, Dimou E, Voutsas E. Separation of the isopropanol-water azeotropic mixture using ionic liquids. Flu Phase Equil. 2018;456:77-83. 
Danielson ND, Mansour FR, Zhou L, Connell CV, Dotlich EM, Gibler JN, Norman BE, Grossman S, Wei W, Zhang Y. Liquid chromatography with alkylammonium formate ionic liquid mobile phases and fluorescence detection. J Chromatogr A. 2018;1559:128-35. https://doi.org/10.1016/j. chroma.2018.03.020.

Fattahi N, Assadi Y, Hosseini MRM, Jahromi EZ. Determination of chlorophenols in water samples using simultaneous dispersive liquid-liquid microextraction and derivatization followed by gas chromatography-electron-capture detection. J Chromatogr A. 2007;1157(1-2):23-9.

Fiamegos YC, Stalikas CD. In-drop derivatisation liquid-phase microextraction assisted by ion-pairing transfer for the gas chromatographic determination of phenolic endocrine disruptors. Anal Chim Acta. 2007;597(1):32-40.

Fu X, Liao Y, Liu J. Sample preparation for pharmaceutical analysis. Anal Bioanal Chem. 2005;381(1):75-7.

Gaillard Y, Prevosto JM, Cheminel V, Soares O, Chaulet JF. New solid-phase extraction for an improved high-performance liquid chromatographic procedure for the quantitation of halofantrine and monodesbutylhalofantrine in blood or plasma. J Chromatogr B. 1995;668(2):315-21.

Jahromi EZ, Bidari A, Assadi Y, Hosseini MRM, Jamali MR. Dispersive liquid-liquid microextraction combined with graphite furnace atomic absorption spectrometry: ultra trace determination of cadmium in water samples. Anal Chim Acta. 2007;585(2):305-11.

Jeevana Jyothy B, Padmaja G. UV spectrophotometric method for estimation of new drug, daclatasvir hydrochloride. Inter Res j of Pharm. 2016;7(9):1-3.

Kozani RR, Assadi Y, Shemirani F, Hosseini MRM, Jamali MR. Part-per-trillion determination of chlorobenzenes in water using dispersive liquid-liquid microextraction combined gas chromatography-electron capture detection. Talanta. 2007;72(2):387-93.

Kramer KE, Andrews ARJ. Screening method for 11-nor-delta9tetrahydrocannabinol-9-carboxylic acid in urine using hollow fiber membrane solvent microextraction with in-tube derivatization. J Chromatogr B. 2001;760:27-36.

Liu Y, Zhao EC, Zhu WT, Gao HX, Zhou ZQ. Determination of four heterocyclic insecticides by ionic liquid dispersive liquid-liquid microextraction in water samples. J Chromatogr A. 2009;1216(6):885-91.

Lucie N, Hana V. A review of current trends and advances in modern bioanalytical methods: chromatography and sample preparation. Anal Chim Acta. 2009;656(1-2):8-35.

Nagaraju D, Huang SD. Determination of triazine herbicides in aqueous samples by dispersive liquid-liquid microextraction with gas chromatography-ion trap mass spectrometry. J Chromatogr B. 2007;1161(1-2):89-97.

Nageswara Rao R, Gangu Naidu C, Suresh CV, Srinath N, Padiya R. Ionic liquid based dispersive liquid-liquid microextraction followed by RP-HPLC determination of balofloxacin in rat serum. Anal Methods. 2014a;6:1674-83.

Nageswara Rao R, Vali RM, Rao AVP. Determination of rifaximin in rat serum by ionic liquid based dispersive liquid-liquid microextraction combined with RP-HPLC. J Sep Sci. 2012;35(15):1945-52.

Nageswara Rao R, Varma NN, Naidu CG. Ionic liquid-based dispersive liquid-liquid microextraction followed by RP-HPLC determination of saquinavir in rat serum: application to pharmacokinetics. Biomed Chrom. 2014b;28(12):1874-80.

Nuhu AA, Basheer C, Saad B. Liquid-phase and dispersive liquid-liquid microextraction techniques with derivatization: recent applications in bioanalysis. J Chromatogr B. 2011;879(17-18):1180-8.

Rahnama Kozani R, Assadi Y, Shemirani F, Hosseini MRM, Jamali MR. Determination of Trihalomethanes in drinking water by dispersive liquidliquid microextraction then gas chromatography with electron-capture detection. Chromatogr. 2007;66(1-2):81-6.

Rezaee M, Assadi Y, Hosseini MRM, Aghaee E, Ahmadi F, Berijani S. Determination of organic compounds in water using dispersive liquid-liquid microextraction. J Chromatogr A. 2006;1116(1-2):1-9.

Rezaee M, Yamini Y, Faraji M. Evolution of dispersive liquid-liquid microextraction method. J Chromatogr A. 2010;1217(16):2342-57.

Rezaee M, Yamini Y, Shariati S, Esrafili A, Shamsipur M. Dispersive liquid-liquid microextraction combined with high-performance liquid chromatography-UV detection as a very simple, rapid and sensitive method for the determination of bisphenol a in water samples. J Chromatogr A. 2009;1216(9):1511-4.

Rykowska I, Ziemblińska J, Nowak I. Modern approaches in dispersive liquid-liquid microextraction (DLLME) based on ionic liquids: a review. J Mol Liq. 2018; 259(1):319-39.

Shokoufi N, Shemirani F, Assadi Y. Fiber optic-linear array detection spectrophotometry in combination with dispersive liquid-liquid microextraction for simultaneous preconcentration and determination of palladium and cobalt. Anal Chim Acta. 2007;597(2):349-56.

Srinivasu G, Nagesh Kumar K, Thirupathi C, Lakshmi Narayana C, Parameswara Murthy C. Development and validation of the chiral HPLC method for daclatasvir in gradient elution mode on amylose-based immobilized chiral stationary phase. Chromatogr. 2016;79(21-22):1457-67.

Suzuki K, Horiba M, Ishikawa K, Katoh S, Naide Y, Yanaoka M, Andoh S. Laboratory and clinical study of Balofloxacin (Q-35), a new fluoroquinolone, in Urinary Tract Infection. Drugs. 1995;49(2):376-8.

Vuckovic D, Zhang X, Cudjoe E, Pawliszyn J. Solid-phase microextraction in bioanalysis: new devices and directions. J Chromatogr A. 2010;1217(25):4041-60.

Yan ZY, Shao XF, Jiang XM, Hu YZ. Studies on the reaction of balofloxacin with bovine serum albumin. Spect and Spec Anal. 2006;26(8):1494-8.

Yao C, Anderson JL. Retention characteristics of organic compounds on molten salt and ionic liquid-based gas chromatography stationary phases. J Chromatogr A. 2009;1216(10):1658-712.

Zeng W, Xu Y, Constanzer M, Woolf EJ. Determination of sitagliptinin human plasma using protein precipitation and tandem mass spectrometry. J Chromatogr B. 2010;878(21):1817-23.

Zhang J, Wu H, Kim E, El-Shourbagy TA. Salting-out assisted liquid/liquid extraction with acetonitrile: a new high throughput sample preparation technique for good laboratory practice bioanalysis using liquid chromatography-mass spectrometry. Biomed Chromatogr. 2009;23(4):419-25.

Zhou QX, Bai HH, Xie GH, Xiao JP. Trace determination of organophosphorus pesticides in environmental samples by temperature-controlled ionic liquid dispersive liquid-phase microextraction. J Chromatogr A. 2008;1188(2):148-53.

Zhou QX, Pang L, Xiao JP. Trace determination of dichlorodiphenyltrichloroethane and its main metabolites in environmental water samples with dispersive liquid-liquid microextraction in combination with high performance liquid chromatography and ultraviolet detector. J Chromatogr A. 2009;1216:6680-4.

\section{Submit your manuscript to a SpringerOpen ${ }^{\circ}$ journal and benefit from:}

- Convenient online submission

- Rigorous peer review

- Open access: articles freely available online

High visibility within the field

- Retaining the copyright to your article

Submit your next manuscript at $>$ springeropen.com 